\title{
Guidewire Breakage: An Unusual Complication of Anterior Odontoid Cannulated Screw Fixation
}

\author{
Tamer Orief, Sharaf Bin-Nafisah, Khaled Almusrea, Mohamed Alfawareh \\ Department of Spine Surgery, Neurosciences Center, King Fahad Medical City, Riyadh, Saudi Arabia
}

\begin{abstract}
The preferred treatment of a type II odontoid fracture is anterior odontoid screw fixation to preserve the cervical spine range of movement. This case report describes an unusual complication of guidewire breakage during anterior odontoid cannulated screw fixation for a 52-year-old patient who presented with a type II odontoid fracture after a motor vehicle accident. The distal segment of the guidewire was bent over the tip of the cannulated odontoid screw and broke off during guidewire withdrawal. The three months follow-up computed tomography examination of the cervical spine showed acceptable screw placement, good odontoid process alignment with incomplete fusion, and no migration of the fractured segment of the guidewire. It is recommended that the guidewire be withdrawn once the cannulated screw is passed through the fractured site into the odontoid process and a new guidewire be used in each surgical procedure instead of been reused to avoid metal stress fatigue that can result in easy breakage.
\end{abstract}

Key Words: Odontoid, Fracture, Complication, Guidewire, Breakage

\section{Introduction}

Odontoid fractures comprise 10 to $15 \%$ of all cervical fractures. The mechanism of injury is a forceful flexion or extension with an axial load. Flexion injuries result in anterior sublaxation, whereas extension injuries result in posterior sublaxation [1].

Anderson and D'Alonzo [2] classified odontoid fractures into three types according to the fracture position. Type I fractures are relatively rare; the fracture line traverses the odontoid tip superior to the transverse ligament. Type II fractures are most common and the fracture line traverses the odontoid base. Type IIa fractures are a subtype where there is communicated fracture of the odontoid process base. Type III fractures include the $\mathrm{C} 2$ body [2]. Type I and III injuries are considered stable and do not require surgical treatment but the most appropriate treatment for type II injuries is controversial.

The conventional approach to stabilize a Type II odontoid fracture has been posterior $\mathrm{C} 1-\mathrm{C} 2$ fusion. An alternative technique of direct screw fixation of the odontoid fracture, which preserves the normal $\mathrm{C} 1-\mathrm{C} 2$ motion, in addition to stabilizing the complex immediately has been advocated. Nakanishi [3] first described the surgical procedure of using anterior lag screw fixation for type II odontoid fractures in 1980 followed by Böhler [4] in 1982, who presented his first series. Dickman et al. [5] described the usefulness of a cannulated screw system for the treatment of upper cervical spine fixation.

However, the anterior approach for direct odontoid process screw fixation has limitations. Patients with short necks and barrel shaped chests might not be surgical candidates for the anterior approach because their anatomy

Received Oct 5, 2010; 1st Revised Nov 5, 2010; Accepted Nov 22, 2010

Corresponding author: Tamer Orief, MSc, MD

Department of Spine Surgery, Neurosciences Center, King Fahad Medical City, MBN 020007,

P. O. Box 59046, Riyadh 11525, Saudi Arabia

Tel: +966-1-288-9999 (Ext.1392), Fax: +966-1-288-9999 (Ext.1391), E-mail: tamerorief@yahoo.com 
results in inadequate screw trajectories. The transverse ligament must be intact, and the fracture line should be in the horizontal plane. Moreover, this anterior technique should be performed only if adequate alignment can be restored before screw insertion. Otherwise, alternative surgical options should be considered [6].

\section{Case Report}

A 52-year-old male was transferred to our hospital with a type II Odontoid fracture secondary to a motor vehicle accident two weeks earlier. The patient complained of severe neck pain upon admission. His neurological examination was unremarkable. Cervical spine radiography and computed tomography (CT) scan revealed an undisplaced type II odontoid fracture (Fig. 1A and 1B). The imaging findings were discussed with the patient as well as conservative and surgical options for treating his unstable odontoid fracture.

The cannulated technique for anterior odontoid screw fixation was preferred. After fibro-optic intubation with inline stabilization, a Mayfield three pins head holder was applied to secure the head. A radiolucent mouth gag was used to keep the mouth open and provide a transoral antero-posterior view. Biplaner fluoroscopy was brought into position. Under fluoroscopic guidance, the patient was placed in the supine position with his neck extended. All attempts were made to minimize the displacement of the odontoid and facilitate the ideal trajectory for screw placement into the apex of the odontoid process. After performing the standard surgical exposure for odontoid screw placement, a transverse cervical skin incision was made over the level of the C5-6 inter-space. The C2-3 level was verified using the lateral C-arm fluoroscopic view. Using fluoroscopy, a 2-mm threaded guidewire was anchored to the midline of the antero-inferior edge of C-2. After anchoring the drill guide and guidewire and confirming the adequate trajectory, the guidewire was then drilled through the fracture line into the distal portion of the fractured dens. The inner guide tube was removed, and the guidewire was left in place. The drill hole was tapped over the guidewire to cut threads in the interface. A $4.0 \times 40 \mathrm{~mm}$ non self-tapping, partially threaded titanium cannulated lag screw was inserted gradually through the guide tube over the guidewire, under direct fluoroscopic view. The distal segment of the guidewire was bent over the tip of the cannulated odontoid screw after being inserted through the fracture line and passed through the most of the distal part of the fractured odontoid process. The distal segment of the guidewire was broken and impacted anteriorly to the odontoid process during attempts of guidewire withdrawal. Screw placement and alignment were confirmed by fluoroscopy. The neck was flexed manually and extended to ensure that the broken segment of the guidewire did not migrate. The wound was closed and a rigid cervical collar was applied. The patient was neurologically intact with an uneventful post-operative course. The patient was followed up monthly in the outpatient clinic. The three month follow-up CT image of the cervical spine revealed proper screw placement and alignment of the odontoid process with incomplete fusion (Fig. 2A, 2B, and
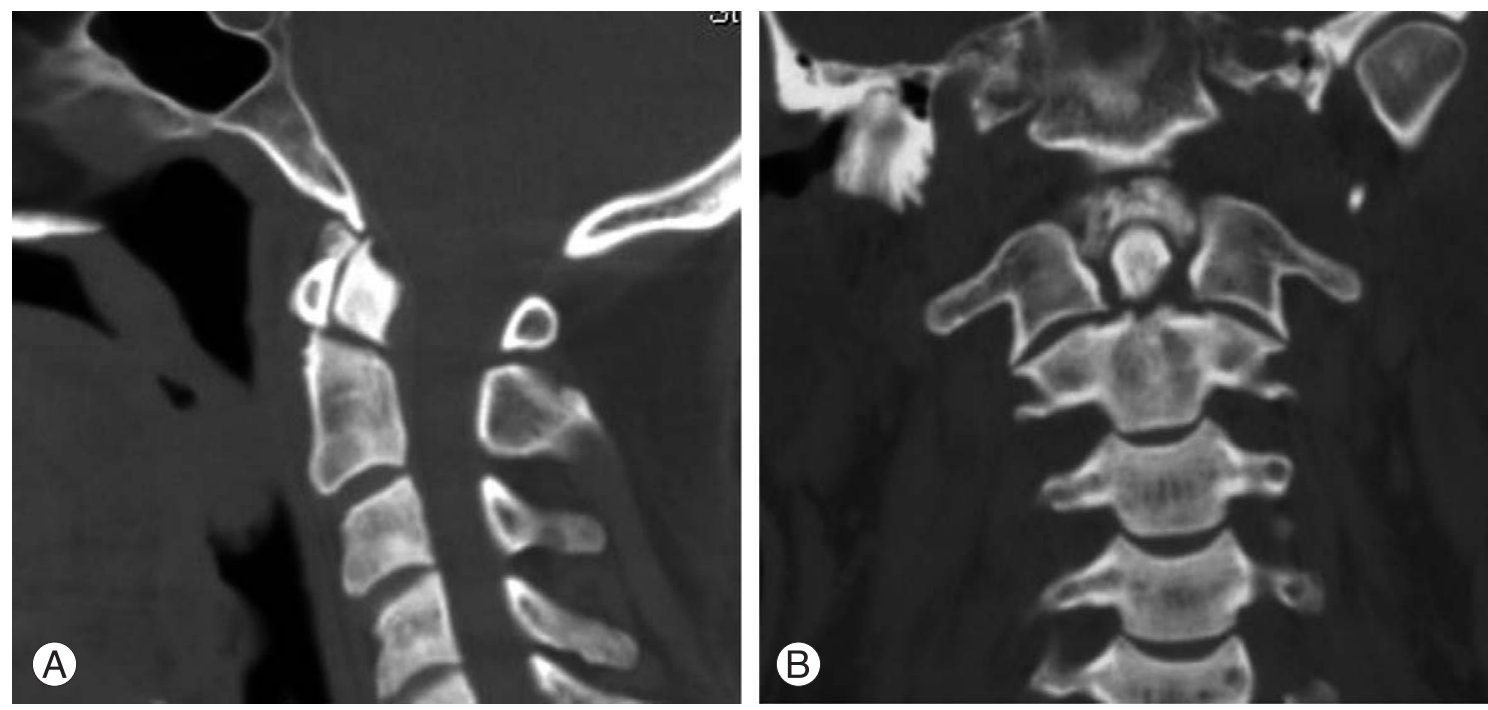

Fig. 1. Pre-operative computed tomography scan of the cervical spine (A, sagittal; B, coronal) showing a non displaced type II odontoid fracture. 
2C). Stability of the odontoid process was demonstrated on a plain X-ray dynamic study of the cervical spine without migration of the fractured segment of the guidewire. (Fig. $3 \mathrm{~A}$ and $3 \mathrm{~B})$

\section{Discussion}

We encountered a case of a 52-year-old patient with a type II odontoid fracture. The optimal treatment varies from conservative management and different types of early surgery. Recent reports, however, have demonstrated unacceptable outcomes after conservative management (e.g., halo immobilization) of type II odontoid fractures [7]. The posterior atlanto-axial fusion approaches are not preferred because the function of the atlanto-axial joint must be sacrificed, which significantly reduces the mobility of the upper
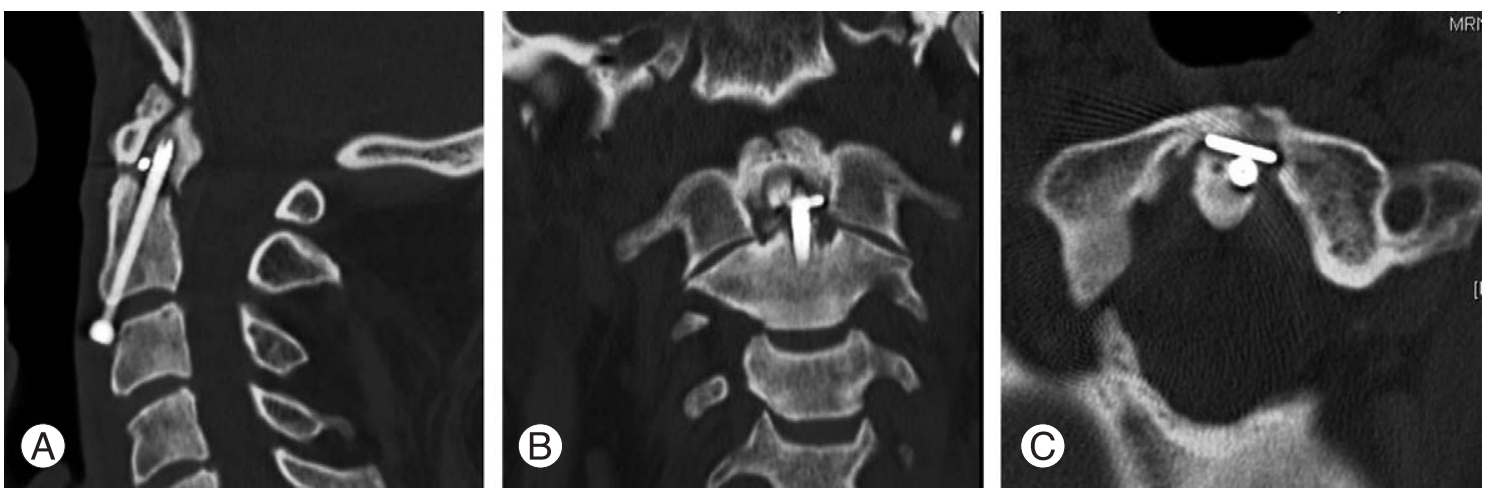

Fig. 2. Three months post-operative computed tomography scan of the cervical spine (A, sagittal; $\mathbf{B}$, coronal; $\mathbf{C}$, axial) showing proper screw placement with incomplete fusion of the odontoid process and no migration of the fractured guidewire.
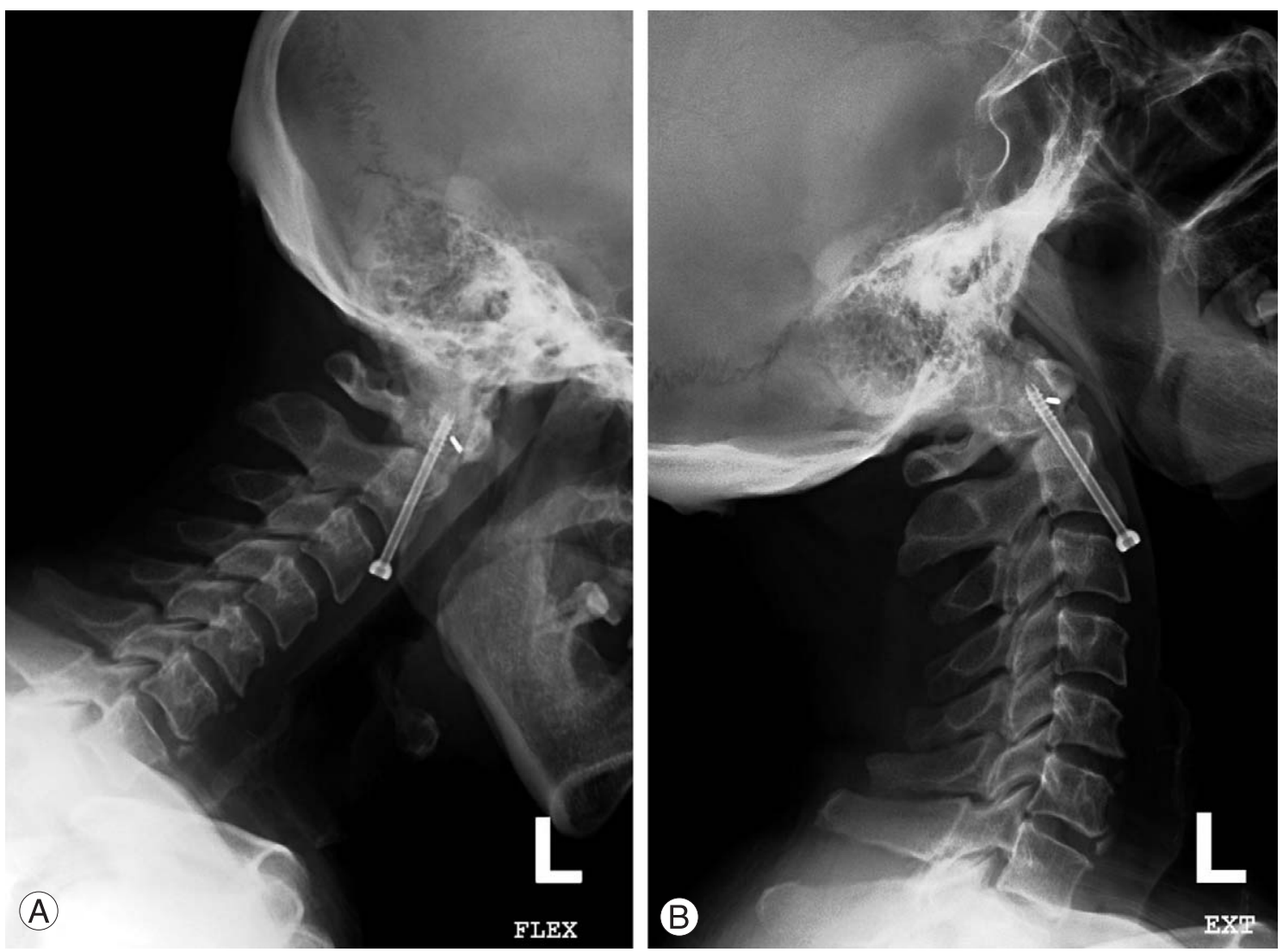

Fig. 3. Three months post-operative X-ray dynamic study of the cervical spine (A, lateral flexion; $\mathbf{B}$, lateral extension) showing acceptable stability and alignment of the odontoid process. 
cervical spine [8].

There are different types of screws are available, including cortical and cancellous bone screws; self-tapping and non self-tapping screws; lag screws or cannulated screws; and fully or partially threaded screws. Cannulated screws have significant advantages over non-cannulated screws for the fixation of odontoid fractures because the guidewire guides the screw position into the bone. The trajectory can be repositioned easily if the original trajectory is not ideal. The guidewire allows continuous fixation of the adjacent unstable bone fragments and prevents migration of unstable bone fragments during screw insertion [5].

In the present case, an unusual complication of guidewire breakage occurred. The distal segment of the guidewire was bent over the tip of the odontoid cannulated screw during screw advancement after passing through the fracture line and most of the distal parts of the odontoid process. Attempts to remove the bent guidewire resulted in the distal end braking off. Any further manipulation to remove or advance the screw was believed to cause more displacement and/or fragmentation of the fractured odontoid process. Intra-operative radiography of the cervical spine on flexion and extension were performed to confirm that the guidewire fragment had not migrated. A broken guidewire fragment cannot be removed surgically without increased iatrogenic tissue trauma to the patient.

The patient was followed up monthly in the outpatient clinic. After three months, the follow-up CT cervical spine revealed proper screw position in the body of $\mathrm{C} 2$ with lateral placement through the odontoid process (Fig. 2C); keeping the fractured odontoid process aligned and close to the body of C2 (Fig. 2A). The stability of the fractured odontoid process over the body of $\mathrm{C} 2$ was demonstrated on a plain X-ray dynamic study of the cervical spine without migration of the fractured segment of the guidewire (Fig. $3 \mathrm{~A}$ and $3 \mathrm{~B}$ ). Acceptable alignment of the odontoid process despite incomplete fusion (Fig. 2A) allowed the patient to be observed for a longer follow-up period (6 months). The cervical spine was immobilized in a neck collar and the possibility of surgery through the posterior approach if incomplete fusion persists was discussed.

The intimacy of bone contact with the implants at six months suggest that despite the use of dissimilar materials in this cannulated screw and guide wire construct, the local environment provided stable implant anchorage as well as acceptable biotolerance [9].

One option to improve the mechanical properties of the guidewire and limit the occurrence of guide wire bending and breakage is to increase the diameter of the guide wire. However, this would mean that all cannulated instruments and implants must also be changed to accommodate a larger guide wire. Moreover, a larger hole in the screwdriver may be required, which can result in the screwdriver tip becoming critically weak.

To avoid breakage of the guidewire during anterior odontoid screw fixation, it is recommended to avoid bending the guidewire during screw advancement, as well as early withdrawal of the guidewire once the cannulated screw is passed through the fractured site and inserted into the proximal part of the fractured odontoid process. A new guidewire should be used in each surgical procedure rather than reusing a guidewire to avoid metal stress fatigue that can result in easily breakage.

\section{REFERENCES}

1. Puttlitz CM, Goel VK, Clark CR, Traynelis VC. Pathomechanisms of failures of the odontoid. Spine (Phila Pa 1976) 2000;25:2868-76.

2. Anderson LD, D'Alonzo RT. Fractures of the odontoid process of the axis. J Bone Joint Surg Am 1974;56:166374.

3. Nakanishi T. Internal fixation of the odontoid fracture. Cent Jpn J Orthop Trauma Surg 1980;23:399-406.

4. Böhler J. Anterior stabilization for acute fractures and nonunions of the dens. J Bone Joint Surg Am 1982;64:18-27.

5. Dickman CA, Foley KT, Sonntag VK, Smith MM. Cannulated screws for odontoid screw fixation and atlantoaxial transarticular screw fixation: technical note. J Neurosurg 1995;83:1095-100.

6. Cannada LK, Turen CH. Indications and techniques of anterior odontoid screw fixation. Curr Opin Orthop 2003;14:182-6.

7. Hadley MN, Dickman CA, Browner CM, Sonntag VK. Acute axis fractures: a review of 229 cases. J Neurosurg 1989;71(5 Pt 1):642-7.

8. Apfelbaum RI. Anterior screw fixation of odontoid fractures. In: Camins MB, O’ Leary PF, editors. Disorders of the cervical spine. Baltimore: Williams and Wilkins; 1992. p. 603-8.

9. Devine DM, Leitner M, Perren SM, Boure LP, Pearce SG. Tissue reaction to implants of different metals: a study using guide wires in cannulated screws. Eur Cell Mater 2009;18:40-8. 